KYUNGPOOK Math. J. 56(2016), 1169-1177

http://dx.doi.org/10.5666/KMJ.2016.56.4.1169

pISSN 1225-6951 eISSN 0454-8124

(c) Kyungpook Mathematical Journal

\title{
On Certain Integral Transforms Involving Hypergeometric Functions and Struve Function
}

\author{
Vijay Kumar Singhal* and Rohit Mukherjee \\ Department of Mathematics, Swami Keshvanand Institute of Technology,Management \\ and Gramothan, Jaipur(Raj.), India \\ e-mail : vijaysinghal01@gmail.com and rohit@skit.ac.in
}

Abstract. This paper is devoted to the study of Mellin, Laplace, Euler and Whittaker transforms involving Struve function, generalized Wright function and Fox's H-function. The main results are presented in the form of four theorems. On account of the general nature of the functions involved here in, the main results obtained here yield a large number of known and new results in terms of simpler functions as their special cases. For the sake of illustration some corollaries have been recorded here as special cases of our main findings.

\section{Introduction}

In 1882, Struve [21] introduced $H_{\nu}$ function as the series solution of the non homogeneous second order Bessel type differential equation. The Struve function has many applications in various fields of physical sciences and also in the fields like electrodynamics, potential theory, optics. Its applications have also been found in water-wave and surface-wave problems, unsteady aerodynamics, distribution of fluid pressure over a vibrating disk. More recently it appeared in particle quantum dynamical studies of spin decoherence ([10], [14]) . Struve function possess power series representation of the form $([4],[22])$.

$$
H_{v}[z]=\sum_{m=0}^{\infty} \frac{(-1)^{m}}{\Gamma\left(m+\frac{3}{2}\right) \Gamma\left(m+v+\frac{3}{2}\right)}\left(\frac{z}{2}\right)^{v+2 m+1}
$$

where $\nu \in \mathrm{R}$ and $\mathrm{z} \in \mathrm{C}$

* Corresponding Author.

Received January 5, 2016; accepted July 6, 2016.

2010 Mathematics Subject Classification: 33C10, 33C60, 33E12 33E20, 33E30, 44A10, $44 \mathrm{~A} 15$.

Key words and phrases: Laplace Transform, Mellin Transform, Euler Transform, Whittaker Transform, Generalized Wright function, Struve function, H-function. 
In 1978, Mathai and Saxena ([9]; see also [1] and [8]), defined H-function by means of the Mellin-Barnes type contour integral as

$$
H_{B, D}^{A, C}[z]=H_{B, D}^{A, C}\left[z \mid \begin{array}{l}
\left(a_{i} ; \alpha_{i}\right)_{1, B} \\
\left(b_{j}, \beta_{j}\right)_{1, D}
\end{array}\right]=\frac{1}{2 \pi i} \int_{L} \Phi(\xi) z^{-\xi} d \xi
$$

where

$$
\Phi(\xi)=\frac{\prod_{j=1}^{A} \Gamma\left(b_{j}+\beta_{j} \xi\right) \prod_{j=1}^{C} \Gamma\left(1-a_{j}-\alpha_{j} \xi\right)}{\prod_{j=A+1}^{D} \Gamma\left(1-b_{j}-\beta_{j} \xi\right) \prod_{j=N+1}^{B} \Gamma\left(a_{j}+\alpha_{j} \xi\right)}
$$

where $\mathrm{L}$ is a suitable contour, the orders $(A, B, C, D)$ are non negative integers such that $1 \leq A \leq D, 0 \leq C \leq B$. The parameters $\alpha_{i}, \beta_{j}$ are positive and $a_{i}, b_{j} i=1,2, \cdots, B ; j=1,2, \cdots, D$ can be arbitrary complex such that $l, l^{\prime}=$ $0,1,2, \cdots ; i=1,2, \cdots, C ; j=1,2, \cdots, A$.

In 1935, Wright [24] introduced the function ${ }_{p} \Psi_{q}(z)$ defined as

$$
{ }_{p} \Psi_{q}(z) \equiv{ }_{p} \Psi_{q}\left[z \mid \begin{array}{c}
\left(a_{i}, \alpha_{i}\right)_{1, p} \\
\left(b_{j}, \beta_{j}\right)_{1, q}
\end{array}\right]=\sum_{k=0}^{\infty} \frac{\prod_{i=1}^{p} \Gamma\left(a_{i}+\alpha_{i} k\right)}{\prod_{j=1}^{q} \Gamma\left(b_{j}+\beta_{j} k\right)} \frac{z^{k}}{k !}
$$

$z \in C, a_{i}, b_{j} \in C$ and $\alpha_{i}, \beta_{j} \in R, i=1,2, \ldots, p ; j=1,2, \ldots, q ; \sum_{j=1}^{q} \beta_{j}-\sum_{i=1}^{p} \alpha_{i}>-1$

This function is called by generalized Wright function [3], [11], [12], [15], [19]. Generalized Wright function generalizes many special functions. In 2002, Kilbas, Saigo and Trujillo studied the properties of the generalized Wright function and proved its integral representation in terms of Mellin Barnes integral [3, Sec 1.19], with a special path of integration. In their paper Kilbas, Saigo and Trujillo [5], established a theorem to represent generalized Wright function in terms of the $\mathrm{H}$-function.

The Mellin transform ([16]; see also [2] and [7]) of $f(x)$ is denoted by

$$
M[f(x), s]=F(s)=\int_{0}^{\infty} x^{s-1} f(x) d x
$$

and the inverse Mellin transform is defined as

$$
f(x)=\frac{1}{2 \pi i} \int_{c-i \infty}^{c+i \infty} F(s) x^{-s} d s
$$


The Laplace transform ([16]; see also [2] and [7]) of $f(x)$ is denoted by

$$
L[f(x)]=F(s)=\int_{0}^{\infty} e^{-s x} f(x) d x
$$

The Euler (Beta) transform ([16]; see also [2] and [7]) of $f(x)$ is denoted by

$$
B\{f(z): a, b\}=\int_{0}^{1} z^{a-1}(1-z)^{b-1} f(z) d z
$$

\section{Main Results}

Theorem 2.1. If

$$
\begin{aligned}
& a_{j}, b_{j} \in C, \alpha_{j}, \beta_{j} \in R, \\
& \sum_{i=1}^{C} E_{i}-\sum_{i=C+1}^{B} E_{i}+\sum_{i=1}^{A} F_{i}-\sum_{i=A+1}^{D} F>0, \sum_{j=1}^{q} \beta_{j}-\sum_{i=1}^{p} \alpha_{i}<1, \\
& |\arg (z)|<\frac{1}{2}\left(\sum_{i=1}^{C} E_{i}-\sum_{i=C+1}^{B} E_{i}+\sum_{i=1}^{A} F_{i}-\sum_{i=A+1}^{D} F_{i}\right) \pi, \\
& -\min _{1 \leq j \leq A} R\left(\frac{f_{j}}{F_{j}}\right)<\operatorname{Re}(s)<\max _{1 \leq j \leq C} R\left(\frac{1-e_{j}}{E_{j}}\right), \\
& \left|\arg \left(-z_{1}\right)\right|<\left(1-\sum_{j=1}^{q} \beta_{j}+\sum_{i=1}^{p} \alpha_{i}\right) \frac{\pi}{2}
\end{aligned}
$$

then

$$
H_{B+q+1, D+p}^{A+p, C+1}\left[-z^{\delta} \mid \begin{array}{c}
(1,1),\left(e_{j}+E_{j}(s+2 m h+h v+h), E_{j} \delta\right)_{1, B},\left(b_{i}, \beta_{i}\right)_{1, q} \\
\left(a_{i}, \alpha_{i}\right)_{1, p},\left(f_{j}+F_{j}(s+2 m h+h v+h), F_{j} \delta\right)_{1, D}
\end{array}\right]
$$

Proof. We first express the generalized Wright function in its integral representation in terms of Mellin Barnes integral [5], then use the expansion (1.1) for the Struve function and the representation (1.2) of the $\mathrm{H}$-function. On interchanging the order of integration and summation and thereafter applying Mellin transform (1.6) we arrive at the desired result. 
Special Cases

(i) Taking

$$
p=q=2 ; a_{1}=\gamma, b_{1}=\eta, b_{2}=\beta, \alpha_{1}=\mu, \beta_{1}=\lambda, \beta_{2}=\alpha, a_{2}=1=\alpha_{2}
$$

in (2.1), we get

Corollary 2.2 .

$$
\begin{array}{r}
\int_{0}^{\infty} x^{s-1} H_{v}\left[a x^{h}\right] E_{\alpha, \beta, \lambda}^{\gamma, \eta, \mu}\left[z_{1} x^{\delta}\right] H_{B, D}^{A, C}\left[z x \mid \begin{array}{l}
\left(e_{j}, E_{j}\right)_{1, B} \\
\left(f_{j}, F_{j}\right)_{1, D}
\end{array}\right] d x \\
=\left(\frac{a}{2}\right)^{v+1} z^{-(s+h(v+1))} \sum_{m=0}^{\infty} \frac{(-1)^{m}}{\Gamma\left(m+\frac{3}{2}\right) \Gamma\left(v+m+\frac{3}{2}\right)}\left(\frac{a}{2}\right)^{2 m} z^{-2 h m} .
\end{array}
$$

$$
\frac{\Gamma(\eta)}{\Gamma(\gamma)} H_{B+3, D+2}^{A+2, C+1}\left[-\frac{z \delta}{z_{1}} \mid \begin{array}{c}
(1,1),\left(e_{j}+E_{j}(s+2 m h+h v+h), E_{j} \delta\right)_{1, B},(\eta, \lambda),(\beta, \alpha) \\
(\gamma, \mu),(1,1),\left(f_{j}+F_{j}(s+2 m h+h v+h), F_{j} \delta\right)_{1, D}
\end{array}\right]
$$

under the conditions derived from those mentioned with Theorem 2.1, where $E_{\alpha, \beta, \lambda}^{\gamma, \eta, \mu}\left[z_{1} x^{\delta}\right]$ is Generalized Mittag leffler function due to Salim and Faraj [13].

(ii) Taking $v=\frac{1}{2}$ in (2.1) we get

\section{Corollary 2.3.}

$$
\begin{array}{r}
\int_{0}^{\infty} x^{s-\frac{h}{2}-1}\left(1-\cos a x^{h}\right)_{p} \Psi_{q}\left[z_{1} x^{\delta} \mid \begin{array}{c}
\left(a_{j}, \alpha_{j}\right)_{1, p} \\
\left(b_{j}, \beta_{j}\right)_{1, q}
\end{array}\right] H_{B, D}^{A, C}\left[z x \mid \begin{array}{c}
\left(e_{j}, E_{j}\right)_{1, B} \\
\left(f_{j}, F_{j}\right)_{1, D}
\end{array}\right] d x \\
=a^{2}\left(\frac{1}{2}\right)^{3 / 2} z^{-\left(s+\frac{3}{2} h\right)} \sum_{m=0}^{\infty} \frac{(-1)^{m}}{\Gamma\left(m+\frac{3}{2}\right) \Gamma(m+2)}\left(\frac{a}{2}\right)^{2 m} z^{-2 h m} . \\
H_{B+q+1, D+p}^{A+p, C+1}\left[-\frac{z}{z}^{\delta} \mid \begin{array}{c}
(1,1),\left(e_{j}+E_{j}\left(s+2 m h+\frac{3 h}{2}\right), E_{j} \delta\right)_{1, B},\left(b_{i}, \beta_{i}\right)_{1, q} \\
\left(a_{i}, \alpha_{i}\right)_{1, p},\left(f_{j}+F_{j}\left(s+2 m h+\frac{3 h}{2}\right), F_{j} \delta\right)_{1, D}
\end{array}\right]
\end{array}
$$

under the conditions derived from those mentioned with Theorem 2.1.

(iii) Taking $v=-\left(n+\frac{1}{2}\right)$, result (2.1) takes the form

\section{Corollary 2.4.}

$$
\begin{array}{r}
\int_{0}^{\infty} x^{s-1} J_{n+\frac{3}{2}}\left(a x^{h}\right)_{p} \Psi_{q}\left[z_{1} x^{\delta} \mid \begin{array}{c}
\left(a_{j}, \alpha_{j}\right)_{1, p} \\
\left(b_{j}, \beta_{j}\right)_{1, q}
\end{array}\right] H_{B, D}^{A, C}\left[z x \mid \begin{array}{l}
\left(e_{j}, E_{j}\right)_{1, B} \\
\left(f_{j}, F_{j}\right)_{1, D}
\end{array}\right] d x \\
=(-1)^{n}\left(\frac{a}{2}\right)^{-\left(n-\frac{1}{2}\right)} z^{-(s-h(n-1 / 2))} \sum_{m=0}^{\infty} \frac{(-1)^{m}}{\Gamma\left(m+\frac{3}{2}\right) \Gamma(m-n+1)}\left(\frac{a}{2}\right)^{2 m} z^{-2 h m} .
\end{array}
$$


$(2.4)$

$$
H_{B+q+1, D+p}^{A+p, C+1}\left[-\frac{z}{z_{1}} \mid \begin{array}{c}
(1,1),\left(e_{j}+E_{j}\left(s+2 m h-n h+\frac{h}{2}\right), E_{j} \delta\right)_{1, B},\left(b_{i}, \beta_{i}\right)_{1, q} \\
\left(a_{i}, \alpha_{i}\right)_{1, p},\left(f_{j}+F_{j}\left(s+2 m h-n h+\frac{h}{2}\right), F_{j} \delta\right)_{1, D}
\end{array}\right]
$$

under the conditions derived from those mentioned with Theorem 2.1.

Theorem 2.5. If $\rho, \lambda, v \in C, R(\rho)>0, R(\lambda)>0, h, k, \delta, \sigma>0,\left|\arg \left(-z_{1}\right)\right|<$

$$
\begin{aligned}
(1- & \left.\sum_{j=1}^{q} \beta_{j}+\sum_{i=1}^{p} \alpha_{i}\right) \frac{\pi}{2} \text { and } \sum_{j=1}^{q} \beta_{j}-\sum_{i=1}^{p} \alpha_{i}<1 \text { then } \\
& \quad \int_{0}^{t} x^{\rho-1}(t-x)^{\lambda-1} H_{v}\left[a x^{h}(t-x)^{k}\right]_{p} \Psi_{q}\left[z_{1} x^{-\delta}(t-x)^{-\mu} \mid \begin{array}{c}
\left(a_{j}, \alpha_{j}\right)_{1, p} \\
\left(b_{j}, \beta_{j}\right)_{1, q}
\end{array}\right] d x \\
= & \left(\frac{a}{2}\right)^{v+1} t^{\rho+\lambda+(h+k)(v+1)-1} \sum_{m=0}^{\infty} \frac{(-1)^{m}}{\Gamma\left(m+\frac{3}{2}\right) \Gamma\left(v+m+\frac{3}{2}\right)}\left(\frac{a}{2}\right)^{2 m} t^{2(h+k) m} .
\end{aligned}
$$

$$
H_{q+3, p+1}^{p, 3}\left[-\frac{t}{z}^{\delta+\mu} \mid \begin{array}{c}
(1,1),(1-\rho-h(2 m+v+1), \delta),(1-\lambda-k(2 m+v+1), \mu),\left(b_{i}, \beta_{i}\right)_{1, q} \\
\left(a_{i}, \alpha_{i}\right)_{1, p},(1-\rho-\lambda-(h+k)(2 m+v+1), \delta+\mu)
\end{array}\right]
$$

Proof. In order to establish the result (2.5), we first express the generalized Wright function in terms of Mellin-Barnes integral [5], then use the expansion (1.1). On interchanging the order of integration and summation and thereafter applying Euler Transform (1.8), we arrive at the desired result.

Special Cases:

(i) Taking $v=-\left(n+\frac{1}{2}\right)$, result (2.5) takes the form

\section{Corollary 2.6.}

$$
\begin{aligned}
& \int_{0}^{t} x^{\rho-1}(t-x)^{\lambda-1} J_{n+\frac{3}{2}}\left[a x^{h}(t-x)^{k}\right]_{p} \Psi_{q}\left[z_{1} x^{-\delta}(t-x)^{-\mu} \mid \begin{array}{c}
\left(a_{j}, \alpha_{j}\right)_{1, p} \\
\left(b_{j}, \beta_{j}\right)_{1, q}
\end{array}\right] d x \\
= & \left(\frac{a}{2}\right)^{-\left(n-\frac{1}{2}\right)} t^{\rho+\lambda-(h+k)\left(n-\frac{1}{2}\right)-1} \sum_{m=0}^{\infty} \frac{(-1)^{m}}{\Gamma\left(m+\frac{3}{2}\right) \Gamma(m-n+1)}\left(\frac{a}{2}\right)^{2 m} t^{2(h+k) m} .
\end{aligned}
$$

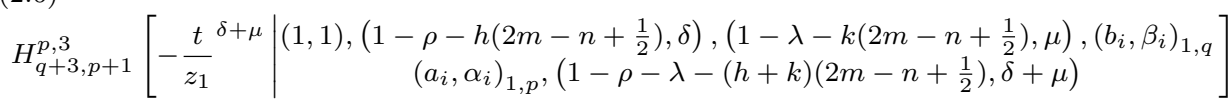

valid under the same conditions derived from those mentioned with Theorem 2.5 .

(ii) On taking $p=1, q=1, a_{1}=\gamma, \alpha_{1}=\kappa, b_{1}=\beta, \beta_{1}=\alpha$, we get 


\section{Corollary 2.7}

$$
\begin{array}{r}
\int_{0}^{t} x^{\rho-1}(t-x)^{\lambda-1} H_{\nu}\left[a x^{h}(t-x)^{k}\right] E_{\alpha, \beta}^{\gamma, \kappa}\left[z_{1} x^{-\delta}(t-x)^{-\mu}\right] d x \\
=\left(\frac{a}{2}\right)^{\nu+1} \frac{t^{\rho+\lambda+(h+k)(\nu+1)-1}}{\Gamma(\gamma)} \sum_{m=0}^{\infty} \frac{(-1)^{m}}{\Gamma\left(m+\frac{3}{2}\right) \Gamma\left(m+\nu+\frac{3}{2}\right)}\left(\frac{a}{2}\right)^{2 m} t^{2(h+k) m} .
\end{array}
$$

$$
H_{4,2}^{1,3}\left[-\frac{t}{z_{1}}{ }^{\delta+\mu} \mid \begin{array}{c}
(1,1),(1-\rho-h(2 m+\nu+1), \delta),(1-\lambda-k(2 m+\nu+1), \mu),(\beta, \alpha) \\
(\gamma, \kappa),(1-\rho-\lambda-(h+k)(2 m+\nu+1), \delta+\mu)
\end{array}\right]
$$

valid under the same conditions derived from those mentioned with Theorem 2.5. Here $E_{\alpha, \beta}^{\gamma, \kappa}[z]$ is the generalized Mittag-Leffler function given by Srivastava and Tomovski $[20]$.

\section{Theorem 2.8. If}

$$
\begin{gathered}
a_{j}, b_{j} \in C ; \alpha_{j}, \beta_{j} \in R ; \sum_{i=1}^{C} E_{i}-\sum_{i=C+1}^{B} E_{i}+\sum_{i=1}^{A} F_{i}-\sum_{i=A+1}^{D} F_{i}>0 ; R(s)>0 ; \delta, \sigma>0, \\
|\arg (z)|<\frac{1}{2}\left(\sum_{i=1}^{C} E_{i}-\sum_{i=C+1}^{B} E_{i}+\sum_{i=1}^{A} F_{i}-\sum_{i=A+1}^{D} F_{i}\right) \pi, R(\rho+1)+\sigma \min _{1 \leq j \leq A} R\left(\frac{f_{j}}{F_{j}}\right)>0 \\
\left|\arg \left(-z_{1}\right)\right|<\left(1-\sum_{j=1}^{q} \beta_{j}+\sum_{i=1}^{p} \alpha_{i}\right) \frac{\pi}{2}
\end{gathered}
$$

and

$$
\sum_{j=1}^{q} \beta_{j}-\sum_{i=1}^{p} \alpha_{i}<1
$$

then

$$
\begin{gathered}
\int_{0}^{\infty} x^{\rho} e^{-s x} H_{v}\left[a x^{h}\right]_{p} \Psi_{q}\left[z_{1} x^{-\delta} \mid \begin{array}{c}
\left(a_{j}, \alpha_{j}\right)_{1, p} \\
\left(b_{j}, \beta_{j}\right)_{1, q}
\end{array}\right] H_{B, D}^{A, C}\left[z x^{\sigma} \mid \begin{array}{l}
\left(e_{j}, E_{j}\right)_{1, B} \\
\left(f_{j}, F_{j}\right)_{1, D}
\end{array}\right] d x \\
=\left(\frac{a}{2}\right)^{v+1} \frac{z^{-\frac{s+h(v+1)}{\sigma}}}{\sigma} \sum_{m=0}^{\infty} \frac{(-1)^{m}}{\Gamma\left(m+\frac{3}{2}\right) \Gamma\left(v+m+\frac{3}{2}\right)}\left(\frac{a}{2}\right)^{2 m} s^{-2 h m} .
\end{gathered}
$$

$$
H_{1,0: q+1, p ; B, D}^{0,1: p, 1 ; A, C}\left[\begin{array}{c}
\left(-z_{1} s\right)^{-\delta} \\
z s^{-\sigma}
\end{array} \begin{array}{c}
(-\rho-h(2 m+v+1) ; \delta, \sigma):(1,1),\left(b_{i}, \beta_{i}\right)_{1, q}:\left(e_{i}, E_{i}\right)_{1, B} \\
-:\left(a_{i}, \alpha_{i}\right)_{1, p}:\left(f_{i}, F_{i}\right)_{1, D}
\end{array}\right]
$$

Proof. Expressing generalized Wright function in terms of Mellin-Barnes type integral [5] and using expressions (1.1) and (1.2), we arrive at the desired result on applying Laplace transform (1.7). 
Special Case

Taking $p=q=2 ; a_{1}=\gamma, b_{1}=\eta, b_{2}=\beta, \alpha_{1}=\mu, \beta_{1}=\lambda, \beta_{2}=\alpha, a_{2}=1=\alpha_{2}$ in (2.8), we get

\section{Corollary 2.9.}

$$
\begin{aligned}
& \int_{0}^{\infty} x^{\rho} e^{-s x} H_{v}\left[a x^{h}\right] E_{\alpha, \beta, \lambda}^{\gamma, \eta, \mu}\left[z_{1} x^{-\delta}\right] H_{B, D}^{A, C}\left[z x^{\sigma} \mid \begin{array}{l}
\left(e_{j}, E_{j}\right)_{1, B} \\
\left(f_{j}, F_{j}\right)_{1, D}
\end{array}\right] d x \\
= & \left(\frac{a}{2}\right)^{v+1} \frac{z^{-\frac{s+h(v+1)}{\sigma}}}{\sigma} \sum_{m=0}^{\infty} \frac{(-1)^{m}}{\Gamma\left(m+\frac{3}{2}\right) \Gamma\left(v+m+\frac{3}{2}\right)}\left(\frac{a}{2}\right)^{2 m} s^{-2 h m}
\end{aligned}
$$

(2.9)

$$
\frac{\Gamma(\eta)}{\Gamma(\gamma)} H_{1,0: 3,2 ; B, D}^{0,1: 2,1 ; A, C}\left[\begin{array}{c}
\left(-z_{1} s\right)^{-\delta} \\
z s^{-\sigma}
\end{array} \begin{array}{c}
(-\rho-h(2 m+v+1) ; \delta, \sigma):(1,1),(\eta, \lambda),(\beta, \alpha):\left(e_{i}, E_{i}\right)_{1, B} \\
-:(\gamma, \mu),(1,1):\left(f_{i}, F_{i}\right)_{1, D}
\end{array}\right]
$$

under the conditions derived from those mentioned with Theorem 2.8, where $E_{\alpha, \beta, \lambda}^{\gamma, \eta, \mu}\left[z_{1} x^{\delta}\right]$ is Generalized Mittag leffler function due to Salim and Faraj [23]

\section{Theorem 2.10. If}

$$
\begin{gathered}
a_{j}, b_{j}, a, \lambda, \mu, \rho, s, z, z_{1} \in C ; \alpha_{j}, \beta_{j} \in R ; \sum_{i=1}^{C} E_{i}-\sum_{i=C+1}^{B} E_{i}+\sum_{i=1}^{A} F_{i}-\sum_{i=A+1}^{D} F>0 \\
\operatorname{Re}(s)>0 ; h, \delta, \sigma>0,|\arg (z)|<\frac{1}{2}\left(\sum_{i=1}^{C} E_{i}-\sum_{i=C+1}^{B} E_{i}+\sum_{i=1}^{A} F_{i}-\sum_{i=A+1}^{D} F_{i}\right) \pi \\
, \operatorname{Re}(\rho)+|\operatorname{Re}(\mu)|+\min _{1 \leq j \leq A} R\left(\frac{f_{j}}{F_{j}}\right)>-\frac{1}{2},\left|\arg \left(-z_{1}\right)\right|<\left(1-\sum_{j=1}^{q} \beta_{j}+\sum_{i=1}^{p} \alpha_{i}\right) \frac{\pi}{2} \\
\text { and } \sum_{j=1}^{q} \beta_{j}-\sum_{i=1}^{p} \alpha_{i}<1 \text { then } \\
\int_{0}^{\infty} x^{\rho-1} e^{-\frac{s x}{2}} H_{v}\left[a x^{h}\right]_{p} \Psi_{q}\left[z_{1} x^{-\delta} \mid \begin{array}{l}
\left(a_{j}, \alpha_{j}\right)_{1, p} \\
\left(b_{j}, \beta_{j}\right)_{1, q}
\end{array}\right] H_{B, D}^{A, C}\left[z x^{\sigma} \mid \begin{array}{l}
\left(e_{j}, E_{j}\right)_{1, B} \\
\left(f_{j}, F_{j}\right)_{1, D}
\end{array}\right] W_{\lambda, \mu}(s x) d x \\
=\left(\frac{a}{2}\right)^{v+1} s^{-\rho-h(v+1))} \sum_{m=0}^{\infty} \frac{(-1)^{m}}{\Gamma\left(m+\frac{3}{2}\right) \Gamma\left(v+m+\frac{3}{2}\right)}\left(\frac{a}{2}\right)^{2 m} s^{-2 h m} .
\end{gathered}
$$

$$
\left.\begin{array}{c}
H_{2,1 ; B, D ; q+1, p}^{0,2 ; A, C ; p, 1}\left[\begin{array}{c}
z s^{-\sigma} \\
-z_{1}^{-1} s^{-\delta}
\end{array} \mid\left(\frac{1}{2}-\mu-\rho-h(2 m+\nu+1) ; \delta, \sigma\right),\left(\frac{1}{2}+\mu-\rho-h(2 m+\nu+1) ; \delta, \sigma\right):\right. \\
(\lambda-\rho-h(2 m+\nu+1) ; \delta, \sigma): \\
\left(e_{j}, E_{j}\right)_{1, B} ;(1,1),\left(b_{i}, \beta_{i}\right)_{1, q} \\
\left(f_{j}, F_{j}\right)_{1, D} ;\left(a_{i}, \alpha_{i}\right)_{1, p},
\end{array}\right]
$$


Proof. To prove result (2.10), we first use expression (1.1), (1.3) and the MellinBarnes integral of the generalized Wright function [5]. Now by changing order of summation and integration and making use of the following result

$$
\int_{0}^{\infty} e^{-t / 2} t^{\nu-1} W_{\lambda, \mu}(t) d t=\frac{\Gamma\left(\frac{1}{2}+\mu+\nu\right) \Gamma\left(\frac{1}{2}-\mu+\nu\right)}{\Gamma(1-\lambda+\nu)}
$$

where $\operatorname{Re}(\nu \pm \mu)>-\frac{1}{2}$.

We arrive at the result (2.10) after a little simplification.

Special Case:

On Taking $A=2, D=2, B=0=C, f_{1}=0, f_{2}=\frac{\xi}{\eta}, F_{1}=1, F_{2}=\frac{1}{\eta}$ in (2.10), we get

\section{Corollary 2.11 .}

$$
\begin{aligned}
& \int_{0}^{\infty} x^{\rho-1} e^{-\frac{s x}{2}} H_{v}\left[a x^{h}\right]_{p} \Psi_{q}\left[z_{1} x^{-\delta} \mid \begin{array}{c}
\left(a_{j}, \alpha_{j}\right)_{1, p} \\
\left(b_{j}, \beta_{j}\right)_{1, q}
\end{array}\right] Z_{\eta}^{\xi}\left[z x^{\sigma}\right] W_{\lambda, \mu}(s x) d x \\
& =\left(\frac{a}{2}\right)^{v+1} \frac{s}{\eta}^{-\rho-h(v+1))} \sum_{m=0}^{\infty} \frac{(-1)^{m}}{\Gamma\left(m+\frac{3}{2}\right) \Gamma\left(v+m+\frac{3}{2}\right)}\left(\frac{a}{2}\right)^{2 m} s^{-2 h m} .
\end{aligned}
$$

$$
\left.\begin{array}{r}
H_{2,1 ; 0,2 ; ; q+1, p}^{0,2 ; 2,0 ; p, 1}\left[\begin{array}{c}
z s^{-\sigma} \\
-z_{1}^{-1} s^{-\delta}
\end{array} \mid\left(\frac{1}{2}-\mu-\rho-h(2 m+\nu+1) ; \delta, \sigma\right),\left(\frac{1}{2}+\mu-\rho-h(2 m+\nu+1) ; \delta, \sigma\right):\right. \\
(\lambda-\rho-h(2 m+\nu+1) ; \delta, \sigma): \\
-;(1,1),\left(b_{i}, \beta_{i}\right)_{1, q} \\
(0,1),\left(\frac{\xi}{\eta}, \frac{1}{\eta}\right) ;\left(a_{i}, \alpha_{i}\right)_{1, p},
\end{array}\right]
$$

valid under the same conditions surrounding (2.10). Here $Z_{\eta}^{\xi}[z]$ is the Krätzel function (See [6], [9] and [18]).

\section{References}

[1] B. L. G. Braaksma, Asymptotic expansions and analytic continuation for a class of Barnes Integrals, Compositio Math., 15(1964), 239-341.

[2] M. M. Dzherbashyan, Integral Transforms and Representations of Functions in the Complex Domain, Nauka, Moscow, 1966(in Russian).

[3] A. Erdélyi (Ed.) et al., Higher Transcendental Functions, Vols. 1-3, McGraw-Hill, New York(1953).

[4] I. S. Gradshteyin and IM. Ryzhik, Table of Integrals, series and products, 6/e, Academic press, New Delhi(2001).

[5] A. Kilbas and M. Saigo, and J. Trujillo, On the generalized Wright function, Fract. Calc. Appl. Anal., 5(4)(2002), 437-460.

[6] E. Krätzel, Integral transformationsof Bessel type, Generalized functions and Operational calculus, Proc. Conf. Verna, 1975, Bulg. Acad. Sci., Sofia(1979), 148-165. 
[7] O. I. Marichev, Handbook of Integral Transforms of Higher Transcendental Functions: Theory and Algorithmic Tables Ellis Horwood and Halsted Press, New York(1983).

[8] A. M. Mathai and H. J. Haubold, Special Functions for Applied Scientists, Springer(2008).

[9] A. M. Mathai and R. K. Saxena, The H-function with Applications in Statistics and Other Disciplines, Wiley East. Ltd., New Delhi(1978).

[10] T. G. Pederson, Variational approach to excitons in carbon nanotubes, Phys. Rev. B, 67(7) (2003), 1-4.

[11] I. Podlubny, Fractional Differential Equations, Acad. Press, San Diego(1999).

[12] A. Prudnikov, Yu. Brychkov and O. Marichev, Integrals and Series, Some More Special Functions, vol. 3, Gordon and Breach, New York(1992).

[13] O. Salim Tariq and W. Faraj Ahmed, A generalization of Mittag-Leffler function and integral operator associated with Fractional calculus, J. Fract. Cal. Appl., Vol.3(2012) No.5, 1-13.

[14] J. Shao and P. Hänggi, Decoherent dynamics of a two level system coupled to a sea of spins, Phys. Rev. Lett., 81(26)(1988), 5710-5713.

[15] A. K. Shukla and J. C. Prajapati, On a generalization of Mittag-Leffler function and its properties, J. Math. Anal. Appl., 336(2007), 797-811.

[16] I. N. Sneddon, The use of Integral Transforms, New Delhi: TMH;1979

[17] H. M. Srivastava, A contour integral involving Fox's H-function, Indian J. Math., 14(1972), 1-6.

[18] H. M. Srivastava, K. C. Gupta and S. P. Goyal, The H-Functions of One and Two Variables with Applications, South Asian Publs., New Delhi(1982).

[19] H. M. Srivastava and B. R. K. Kashyap, Special Functions in Queuing Theory and Related Stochastic Processes, Acad. Press, New York(1981).

[20] H. M. Srivastava and Z. Tomovski, Fractional calculus with an integral operator containing a generalized Mittag-Leffler function in the kernel, Appl. Math. Comput., Vol.211(2009), 198-210.

[21] H. Struve, Beitrag zur Theorie der Diffraction an Fernrohren, Ann. Physik Chemie, 17(1882), 1008-1016.

[22] G. N. Watson, A treatise on the theory of Bessel functions, 2nd ed., Cambridge univ. press, London 1966.

[23] A. Wiman, Uber den fundamental satz in der theory der funcionen, Acta Math., Vol.29(1905),191-201.

[24] E. M. Wright, The asymptotic expansion of the generalized hypergeometric Function, London Math. Soc., 10(1935), 257-260. 